\title{
Phase dependency of long-term potentiation induction during the intermittent bursts of carbachol-induced $\beta$ oscillation in rat hippocampal slices
}

\author{
Motoshi Nishimura ${ }^{1}$, Hiroki Nakatsuka ${ }^{1}$ and Kiyohisa Natsume ${ }^{1,2}$ \\ ${ }^{1}$ Department of Brain Science and Engineering, Graduate School of Life Science and Systems Engineering, Kyushu Institute of Technology, \\ 2-4 Hibikino, Wakamatsu-ku, Kitakyushu, Fukuoka 808-0196, Japan \\ ${ }^{2}$ Biomedical Informatics R\&D Center, Kyushu Institute of Technology, 2-4 Hibikino, Wakamatsu-ku, Kitakyushu, Fukuoka 808-0196, Japan
}

Received September 18, 2012; accepted November 6, 2012

The rodent hippocampus possesses theta $(\theta)$ and beta ( $\beta)$ rhythms, which occur intermittently as bursts. Both rhythms are related to spatial memory processing in a novel environment. $\theta$ rhythm is related to spatial memory encoding process. $\beta$ rhythm is related to the match/mismatch process. In the match/mismatch process, rodent hippocampus detects a representation matching sensory inputs of the current place among the retrieved internal representations of places. Long-term synaptic potentiation (LTP) is induced in both processes. The cholinergic agent carbachol induces intermittent $\theta$ and $\beta$ oscillations in in vitro slices similar to in vivo bursts. LTP is facilitated during the generation of $\theta$ oscillation, suggesting that the facilitation of LTP is dependent upon the phases of intermittent burst (burst phases) of the oscillation. However, whether this is the case for $\beta$ oscillation has not yet been studied. In the present study, LTP-inducing $\theta$-burst stimulation was administered at the different burst phases of carbacholinduced $\beta$ oscillations (CIBO), and the synaptic changes were measured at $\mathrm{CA3}-\mathrm{CA} 3$ pyramidal cell synapses (CA3 synapse) and at CA3-CA1 pyramidal cell synapses (CA1 synapse). At the CA3 synapse, the largest magnitude of LTP was induced at the late burst phases of CIBO. At the CA1 synapse, LTP was induced only at the late burst phases. Modulation of LTP was suppressed

Corresponding author: Kiyohisa Natsume, Department of Brain Science and Engineering, Graduate School of Life Science and Systems Engineering, Kyushu Institute of Technology, 2-4 Hibikino, Wakamatsuku, Kitakyushu, Fukuoka 808-0196, Japan.

e-mail: natume@brain.kyutech.ac.jp when CIBO was blocked by the application of atropine at both synapses. The results suggest that the bursts of hippocampal $\beta$ rhythm can determine the optimal temporal period for completing with the match/mismatch process.

Key words: hippocampus, $\beta$ rhythm, novelty, match/mismatch process, learning

Rodents have theta $(\theta, 4-8 \mathrm{~Hz})^{1}$ and beta $(\beta, 12-35 \mathrm{~Hz})^{2}$ rhythms associated with the development of spatial memories. When a rodent is placed in a novel environment and begins to explore, hippocampal $\theta$ rhythm is induced initially, followed by $\beta$ rhythm $^{2}$. $\beta$ rhythm is induced independently with the generation of $\theta$ rhythm and both rhythms appear intermittently as bursts in a wax-and-waning fashion. $\theta$ rhythm is thought to be related to spatial memory encoding and retrieval, and $\beta$ rhythm is thought to be related to the match/mismatch process in novelty detection. In the match/mismatch process, the hippocampus discards mismatched place representations and identifies matching representations among those retrieved by incoming sensory inputs of the current place when a rodent revisits a previously experienced place ${ }^{3}$. After the matching, the matched representations are reconsolidated. Memory encoding and matching processes require synaptic plasticity ${ }^{3,7,8} . \beta$ rhythm is also related to the retrieval process in odor discrimination learning ${ }^{4,5}$ and is important in long-range synchronization of cognitive processes ${ }^{6}$.

Long-term synaptic potentiation (LTP) is the foremost 
example of synaptic plasticity as the basis of memory processing and formation. While LTP is observed in in vivo $\theta$ rhythm $^{9}$, it has not yet been examined in $\beta$ rhythm. The cholinergic agent carbachol induces $\theta$ oscillation in rat hippocampal slices ${ }^{10}$, which is similar to in vivo $\theta$ rhythm; in both cases, the oscillation occurs intermittently as bursts ${ }^{11}$. Natsume and Kometani ${ }^{12}$ studied the effect of $\theta$ oscillation generation on LTP and found that the magnitude of LTP is modulated by the timing of the stimulus delivered during the intermittent burst interval. LTP is facilitated when it is induced during $\theta$ rhythm ${ }^{12}$, suggesting that spatial memory encoding and retrieval will occur during the generation of $\theta$ rhythm.

Hippocampus CA3 pyramidal cells have inputs from the dentate gyrus and entorhinal cortex, as well as from other CA3 pyramidal cells. CA1 pyramidal cells have inputs from the entorhinal cortex and CA3 pyramidal cells via Schaffer collaterals $^{13}$. LTP is induced at CA3-CA3 pyramidal cell synapses (CA3 synapse) and at CA3 Schaffer collateral and CA1 pyramidal cell synapses (CA1 synapse) ${ }^{14}$. The hippocampal CA3 region has been proposed to play a role in encoding memories and in the retrieval of encoded representations ${ }^{15}$. The hippocampal CA1 region has been proposed to be involved in the match/mismatch process ${ }^{7,8}$ by comparing the retrieved representations from the CA3 region with afferent sensory inputs from the entorhinal cortex, and identifies matched representations associated with the sensory inputs.

Carbachol-induced $\beta$ oscillation (CIBO) in rat hippocampal slices ${ }^{16,17}$ is a model for in vivo $\beta$ rhythm ${ }^{2}$. CIBO occurs in $\mathrm{CA} 3$ and CA1 regions intermittently as bursts. The relationship between the induction of LTP and $\beta$ rhythm has not yet been established. The magnitude of LTP may be modulated at the different timing of the LTP-inducing stimulus delivered during the intermittent burst interval of CIBO, similar to what is seen in carbachol-induced $\theta$ oscillation. Note that there are two kinds of periodic events in this phenomenon: one is the $\beta$ oscillation and the other is the intermittent burst of $\beta$ oscillation. Here, the "burst phase" is defined as the phase of the intermittent bursts of CIBO, and it is different from the phase of $\beta$ oscillation. In the present study, $\theta$-burst stimulation (TBS), an LTP-inducing stimulus, was given at the various burst phases of CIBO at CA3 and CA1 synapses to clarify whether the magnitude of LTP was modulated or not when CIBO was induced in a hippocampal slice.

\section{Materials and Methods}

The present data were obtained from 300 hippocampal slices $(450-\mu \mathrm{m}$ thick) of 165 male Wistar rats aged 3-5 weeks. The experiments were carried out in compliance with the Guide for the Care and Use of Laboratory Animals at the Graduate School of Life Science and System Engineering of Kyushu Institute of Technology. Rats were anes-

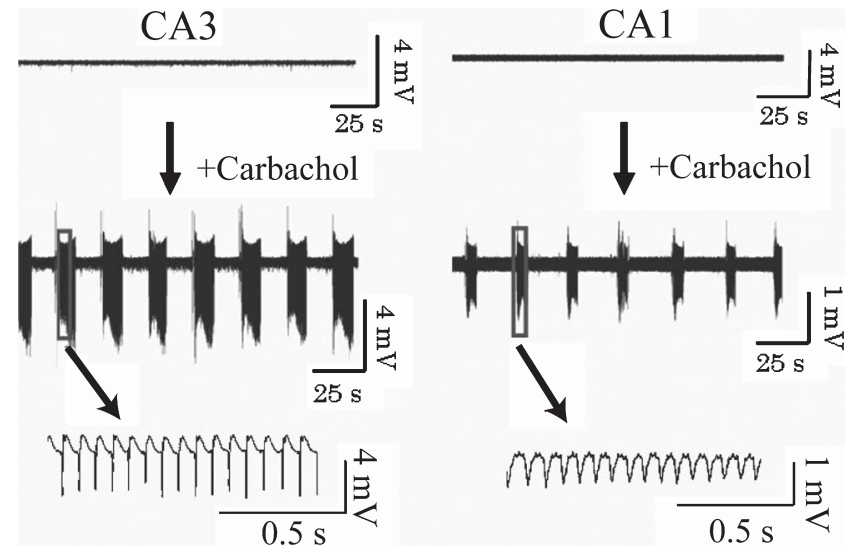

Figure 1 A typical example of CIBO in CA3 and CA1 synapses. Control slices had no spontaneous activity (top). Carbachol-induced bursts of CIBO in CA3 and CA1 regions (middle). The square areas on the left side of the middle figures are expanded in the bottom figures. $\mathrm{CIBO}$ in $\mathrm{CA} 3$ and $\mathrm{CA} 1$ regions in the figure were not measured simultaneously.

thetized by diethyl ether and decapitated. The brains were rapidly removed and placed into oxygenated cold $\left(0^{\circ} \mathrm{C}\right)$ artificial cerebrospinal fluid (ACSF). The composition of ACSF was as follows (in $\mathrm{mM}$ ): $124 \mathrm{NaCl}, 5 \mathrm{KCl}, 1.25$ $\mathrm{NaH}_{2} \mathrm{PO}_{4}, 2 \mathrm{MgSO}_{4}, 26 \mathrm{NaHCO}_{3}, 10$ glucose, and $2 \mathrm{CaCl}_{2}$. The hippocampus was dissected free and transverse slices were obtained using a tissue slicer (Micro Slicer Zero-1, Dosaka-EM Co., Japan). Experimental procedures began after incubation in a holding chamber at room temperature for more than 1 hour.

The cholinergic agent carbachol induces CIBO intermittently in hippocampal slices ${ }^{16}$. The recording electrode $(2 \mathrm{M} \mathrm{NaCl},<2 \mathrm{M} \Omega)$ was placed in the stratum pyramidale in $\mathrm{CA} 3$ or CA1 subregions to record the CIBO. The field potential of CIBO from the glass microelectrode was amplified $(\times 1000)$, band-pass filtered between $1 \mathrm{~Hz}$ and $0.3 \mathrm{kHz}$ using an extracellular amplifier (ER-1, Cygnus Technology, USA), and sampled on a PC with $1 \mathrm{kHz}$ using pClamp 10.0 software (Molecular Devices Co., USA).

The bursts of CIBO were induced with the application of $30 \mu \mathrm{M}$ carbachol as shown in Figure 1. They achieved steady state approximately $15 \mathrm{~min}$ after the application began. The averaged frequency, amplitude, duration and inter-burst interval were measured in the steady state. The frequency of CIBO within an individual burst reached a steady state $2 \mathrm{~s}$ after the onset of the burst. Frequency was measured by fast Fourier transform (FFT) analysis for $1 \mathrm{~s}$ in the steady state. The analysis was calculated using pClamp 10.0. The duration represents the time that the burst of CIBO was maintained (Fig. 2A). The averaged peak-to-peak amplitude was measured for the same time period for frequency. The interburst interval (IBI) was measured as the time between the end of one burst and the start of the next (Fig. 2A). The bursts of CIBO are simultaneously observed at CA3 and CA $1^{16}$. 
A

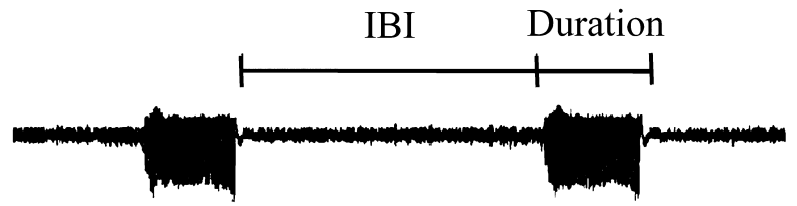

B

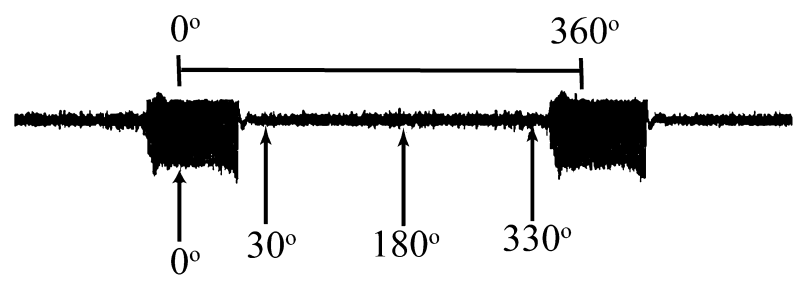

Figure 2 Oscillatory parameters and the burst phases in CIBO. (A) Bursts of carbachol-induced $\beta$ oscillations (CIBO). The duration and inter-burst interval (IBI) of CIBO were defined. (B) The burst phases at which $\theta$ burst stimulation (TBS) was administered are defined as shown in CIBO.

CA3 and CA1 LTP experiments were done. In the CA3 experiment, concentric bipolar stimulation electrodes with external diameters of $300 \mu \mathrm{m}$ (FHC Inc., USA) were placed in the stratum radiatum to stimulate associational fibers of CA3 pyramidal neurons antidromically; a recording electrode was placed in the stratum radiatum to record the population excitatory postsynaptic potential (pEPSP) at the CA3CA3 pyramidal cell synapses (called CA3 synapse). In the CA1 LTP experiment, the stimulation electrode was put in the stratum radiatum of CA1 to stimulate Schaffer collaterals orthodromically. The recording electrode was placed in the stratum radiatum to record $\mathrm{pEPSP}$ at the CA3-CA1 pyramidal cell synapses (called CA1 synapse). The pEPSP was amplified $(\times 1000)$, band-pass filtered between $1 \mathrm{~Hz}$ and $3 \mathrm{kHz}$ using the extracellular amplifier ER-1 (Cygnus Technology), and sampled on a PC with $10 \mathrm{kHz}$ using pClamp software.

A $100-\mu$ s rectangular test pulse was emitted 3 times at 30 -s intervals around $1 \mathrm{~min}$ before TBS, and 3 times at the same interval around 10,20,30, and $40 \mathrm{~min}$ after TBS to measure the effect of TBS on pEPSP slopes. Test pulses were given at the burst phase of $60^{\circ}$ (Fig. 2B; defined below) of CIBO. The pEPSP slope was calculated from each pEPSP, and the averaged slope among three pEPSPs was plotted at each time point (for example, the figures on the right in Fig. 4). The test pulse began 20 min after the onset of carbachol application. Stimulation intensity of the pulse was adjusted before the application of carbachol so that the amplitude of pEPSP was two-thirds of the maximum amplitude. The stimulation intensity of TBS was also set to the same as that of the test pulse. To validate the induction of LTP, pEPSP was analyzed by measuring its initial slope, which contained only an $\alpha$-amino-3-hydroxy-5-methyl-4isoxazolepropionic acid (AMPA) receptor-dependent fast pEPSP component. The slope was calculated by the least squared method using pClamp. The relative pEPSP slope was defined as that normalized by the averaged pEPSP slope at $1 \mathrm{~min}$ before TBS. The relative pEPSP slopes at $40 \mathrm{~min}$ were statistically compared with those at $1 \mathrm{~min}$ before TBS, and the significant difference probability was calculated to determine whether LTP was induced or not. The magnitude of LTP was defined as the relative pEPSP slope at $40 \mathrm{~min}$ after TBS. TBS, which consisted of 5 bursts of five 10 -ms interval pulses with 200 -ms intervals, was applied. TBS was administered during a burst of CIBO, just after the termination of the CIBO burst, during the rest of the CIBO burst, and just before the onset of the CIBO burst. For the sake of convenience, these TBS stimulus timings are defined as the burst phases of $0^{\circ}, 60^{\circ}, 180^{\circ}$, and $330^{\circ}$, respectively (Fig. 2B). At $2 \mathrm{~s}$ from the onset of CIBO, the frequency reached a steady state as described before, and the burst phase of $0^{\circ}$ is defined as $2 \mathrm{~s}$ after the onset of a burst of CIBO. The burst phase of $360^{\circ}$ is defined as $2 \mathrm{~s}$ after the onset of the next burst of CIBO. The interval between $0^{\circ}$ and $360^{\circ}$ was divided proportionally, and the phases $60^{\circ}, 180^{\circ}$, and $330^{\circ}$ were determined (Fig. 2B). Note again that the "burst phases" are different from the phases determined during periodic $\beta$ oscillation.

Atropine sulfate (muscarinic acetylcholine receptor antagonist; atropine) was applied to determine whether CIBO modulates synaptic plasticity or not. In the pharmacological experiments, atropine was administered $20 \mathrm{~min}$ after the application onset of carbachol and LTP was induced $30 \mathrm{~min}$ later.

All drugs were purchased from Sigma (USA). Data are expressed as mean \pm SEM (standard error of the mean). Statistical significance was set at $\mathrm{p}<0.05$.

\section{Results}

\section{Induction of CIBO}

Hippocampal slices had no spontaneous activity at CA3 and CA1 before the application of carbachol (Fig. 1, top). After the application of carbachol, CIBOs were induced at both regions. The bursts of CIBO occurred simultaneously at $\mathrm{CA} 3$ and $\mathrm{CA} 1$ as reported previously ${ }^{16}$. The frequency of CIBO was $15.1 \pm 0.7 \mathrm{~Hz}$, IBI was $24.9 \pm 2.7 \mathrm{~s}$, duration was $6.8 \pm 0.2 \mathrm{~s}$, and amplitude was $4.5 \pm 0.6 \mathrm{mV}(\mathrm{n}=6)$.

\section{pEPSP recorded in CIBO}

With the application of carbachol, the mono-phase negative deflection that reflects pEPSP sometimes includes positive deflections reflecting population spikes at $\mathrm{CA} 3$ and CA1 synapses. The pEPSPs at both synapses are shown in Figure 3 . To identify pEPSPs, $10 \mu \mathrm{M}$ of the AMPA receptor antagonist 6-cyano-7-nitroquinoxaline-2,3-dione (CNQX) was applied, and pEPSPs were suppressed (Fig. 3). Similar results were observed in 3 slices. The field potential from 2 to $15 \mathrm{~ms}$ after the onset of the stimulation is defined as the duration of pEPSP. 


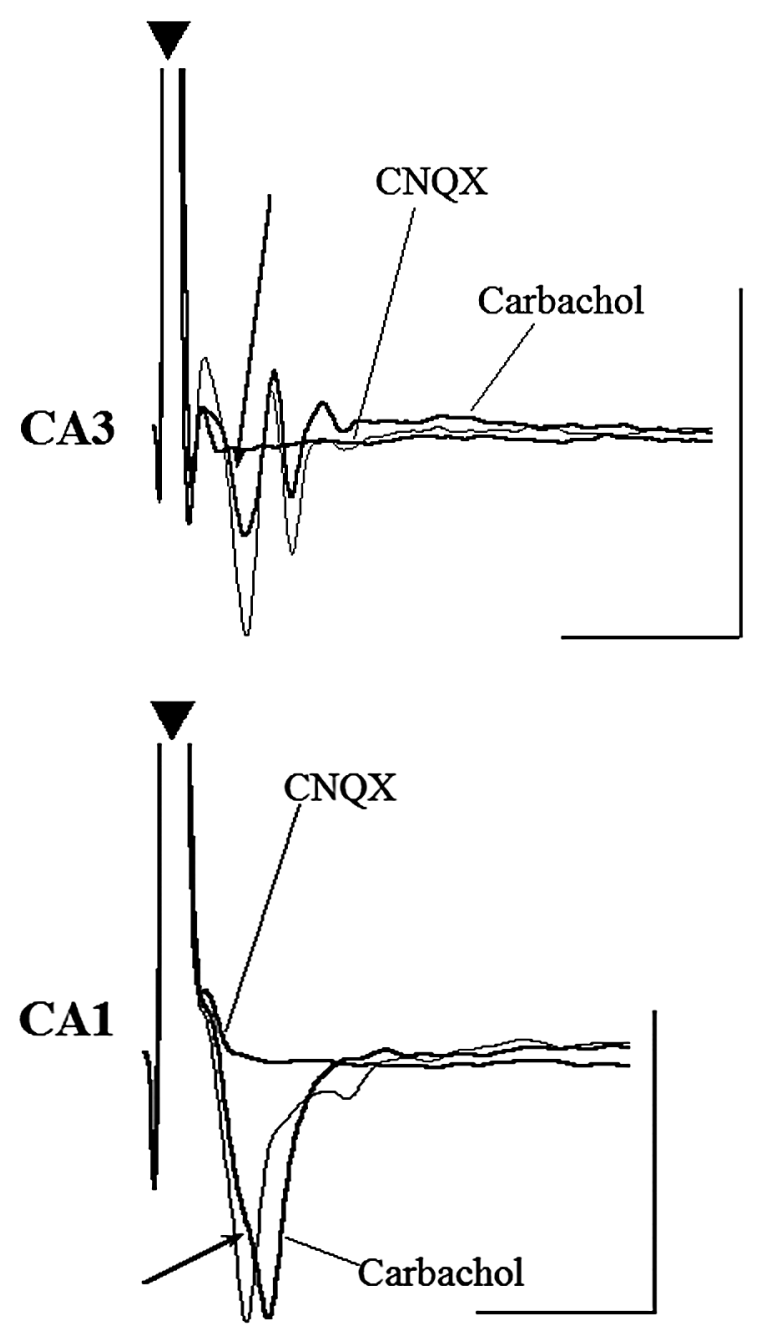

Figure 3 The suppression of population excitatory postsynaptic potential (pEPSP) recorded at CA3 and CA1 synapses by the application of 6-cyano-7-nitroquinoxaline-2, 3-dione (CNQX) in the generation of CIBO. pEPSPs before and after the application of CNQX are shown. Arrows indicate the pEPSP slopes before the application. The inverted black triangles indicate stimulation artifacts. The disconnections of the stimulation artifact in the figure are due to the magnification of the pEPSP slope. Fine line traces indicate pEPSPs before the application of carbachol

\section{Time course of pEPSP without TBS recorded in CIBO}

The slope of pEPSP was calculated between 3 and $10 \mathrm{~ms}$ after the onset of stimulation. The slope at the CA3 synapse was $-0.72 \pm 0.14 \mathrm{mV} / \mathrm{ms}(\mathrm{n}=10)$ and the slope at the CA1 synapse was $-2.7 \pm 0.34 \mathrm{mV} / \mathrm{ms}(\mathrm{n}=10)$ before the application of carbachol. The application of carbachol decreased the slopes as previously reported ${ }^{15,18,19}$. The pEPSP slopes after the application were normalized with those averaged for $1 \mathrm{~min}$ before it. The normalized pEPSP slopes were $0.34 \pm 0.06(n=5)$ at the CA3 synapse, and were $0.36 \pm 0.05$ $(\mathrm{n}=5)$ at the CA1 synapse $20 \mathrm{~min}$ after the application. The decrease was significant at both synapses (paired $t$-test; $\mathrm{p}<0.05)$.
pEPSPs were recorded with test pulses for 41 min every 10 min without TBS, as in the LTP experiment protocol. The relative pEPSP slope at the CA3 synapse at the last $1 \mathrm{~min}$ to that at the first 1 min was $0.86 \pm 0.06(\mathrm{n}=3)$, and that at the CA1 synapse was $0.81 \pm 0.13(n=3)$. They were not significantly different (paired $t$-test with those at the first $1 \mathrm{~min} ; \mathrm{p}>0.05 ; \mathrm{n}=3$ at each synapse). Thus, the slope did not change significantly during the period of the LTP experiment.

\section{Synaptic plasticity induced with TBS at different burst phases of CIBO}

When TBS was administered at the $0^{\circ}$ burst phase, the relative pEPSP slope significantly increased and was maintained for at least $40 \mathrm{~min}$ at the CA3 synapse (Fig. 4A). Thus, LTP was induced (Mann-Whitney U test compared with the relative $p E P S P$ slopes before TBS, $p<0.01$ ). At the CA1 synapse, TBS increased the pEPSP slope just after the stimulation. Subsequently, the slope decreased. TBS did not have a long-term effect and induced short-term potentiation (Fig. 4A; one-way repeated measures ANOVA with Bonferroni's post-hoc test, $\mathrm{p}<0.01$ ). TBS at the $60^{\circ}$ burst phase at the CA3 synapse induced LTP (Fig. 4B; Mann-Whitney U test, $\mathrm{p}<0.05)$. TBS did not significantly induce LTP or LTD at the same burst phases at the CA1 synapse. When TBS was administered at the $180^{\circ}$ burst phase, LTP was induced at both the CA3 and CA1 synapses (Fig. 4C). The magnitudes of LTP were significantly different from baseline (Mann-Whitney U test; $\mathrm{p}<0.01$ ). When TBS was administered at the $330^{\circ}$ burst phase, LTPs were also induced at both the CA3 and CA1 synapses (Fig. 4D). The magnitudes of LTP at both synapses were significantly increased (Mann-Whitney U test; $\mathrm{p}<0.01$ ).

Figure 5 (top) summarizes TBS-induced LTP at all burst phases of CIBO at the CA3 synapse. The magnitudes of LTP at all phases were significantly different from baseline. The magnitudes at the late burst phases were larger than those at the earlier phases. At the CA1 synapse, TBS induced significant LTP only at the later burst phases of $180^{\circ}$ and $330^{\circ}$ of CIBO (Fig. 5, bottom). In contrast to what is seen in carbachol-induced $\theta$ oscillation, in $\beta$ oscillation, the magnitude of LTP at the rest phase of the oscillation is facilitated at the CA3 synapse and LTP is induced at the CA1 synapse.

Pharmacological experiment of the synaptic plasticity study CIBO is sensitive to atropine, as has been previously reported $^{16,17}$. Atropine suppressed CIBO in CA3 and CA1 synapses, and induced individual field activities (IFA) ${ }^{16}$ in both regions (Fig. 6). The induction of LTP was investigated during the generation of IFA. The protocol to induce LTP was identical to that described previously, except that TBS was not administered at the specific burst phase shown in Figure 5 because the application of atropine had suppressed the CIBO bursts, and the burst phase was not determined. 
A

CA3

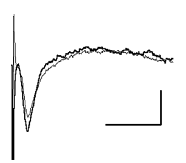

CA1
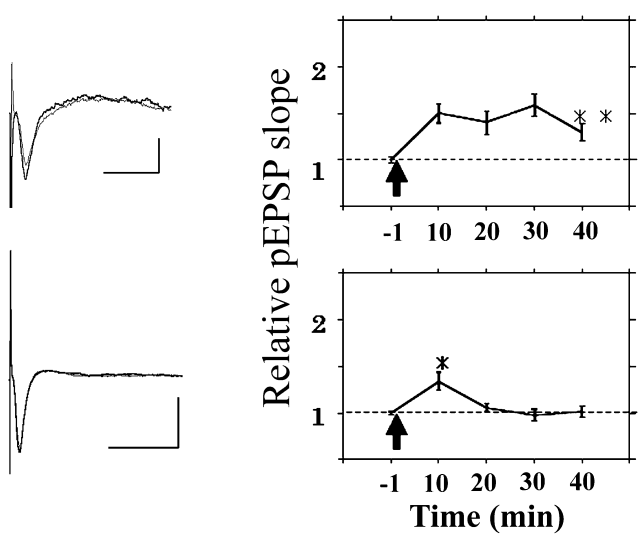

C

CA3

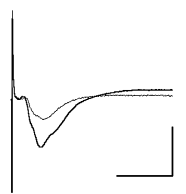

CA1

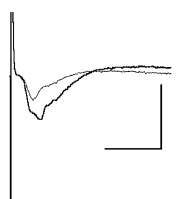

B

CA3

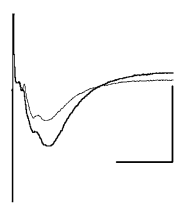

CA1
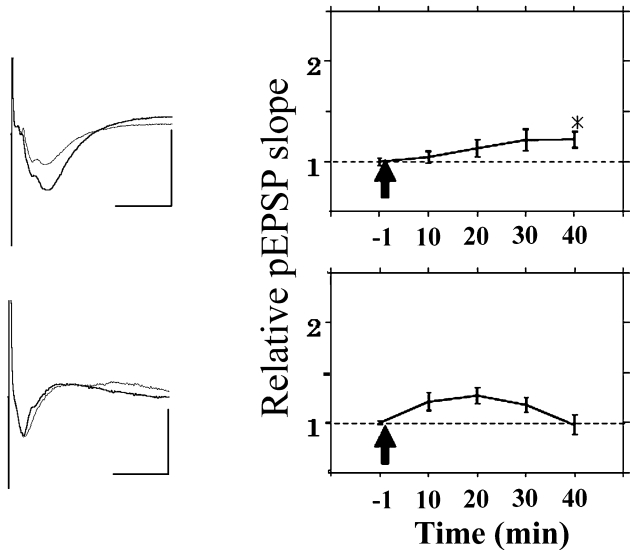

D

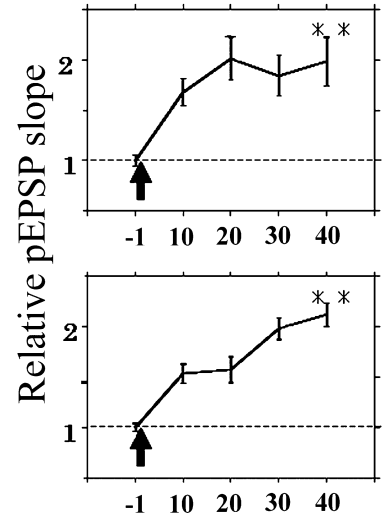

Time (min)

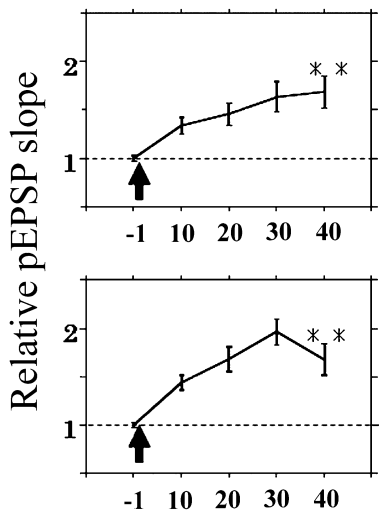

Time (min)

Figure 4 The time courses of relative pEPSP slope with TBS at the different burst phases in CIBO. The synaptic change by TBS administered at the burst phase $0^{\circ}(\mathrm{A})$, the $60^{\circ}$ burst phase in CIBO (B), the $180^{\circ}$ burst phase (C) and the $330^{\circ}$ burst phase (D) at the CA3 and CA1 synapses. The time courses of relative pEPSP slopes are on the right column. The thick black arrows indicate the onset of TBS. Minus 1 min on the x-axis indicates 1 min before TBS. Data on the right at CA3 and CA1 synapses were obtained from 7, and 4 slices, respectively (A), from 9 , and 4 slices, respectively (B), from 6, and 4 slices, respectively (C) and from 6 , and 5 slices, respectively (D). In the left column, the representative pEPSPs are shown. Fine and thick traces are pEPSPs before and after TBS, respectively. Scale bars; $20 \mathrm{~ms}$ and $3 \mathrm{mV}$ in all figures. pEPSP slopes at $40 \mathrm{~min}$ are compared with those at -1 min. ${ }^{*} p<0.05$ and ${ }^{* *} p<0.01$.

While we attempted to induce synaptic change by TBS at the CA 3 and CA1 synapses in 3 hippocampal slices, LTP was induced in neither region (Fig. 6). The magnitude of LTP at the CA3 and CA1 synapses were $1.08 \pm 0.06(n=3)$ and $0.95 \pm 0.04(\mathrm{n}=3)$, respectively (Fig. 6A and B). They were not significantly different from respective baselines. LTP was not induced with TBS under the suppression of the CIBO burst. However, there is a possibility that the maximum pEPSP slope changes with the application of atropine; thus, there would be less room for the detection of an increase in the pEPSP slope. Therefore, the maximum pEPSP slope was measured before TBS application. The ratio of the test pEPSP slope to the maximum slope was calculated. The ratios were $37 \pm 7 \%(n=3)$ and $34 \pm 5 \%(n=3)$ at the CA3 and CA1 synapses, respectively. Thus, there was adequate room for the pEPSP slope to be increased by TBS and the possibility was ruled out.

\section{Dependence of the relative pEPSP slope on the burst phases of CIBO}

pEPSP is dependent upon the phase of the $\theta$ rhythm $^{20}$. There is a possibility that pEPSP changes with the burst phases of CIBO, and that these changes lead to different magnitudes of LTP with TBS ${ }^{21,22}$ at different burst phases, as shown in Figures 4 and 5. In the LTP experiment, TBS was administered at the different burst phases of CIBO. TBS consisted of 5 bursts of 5 pulses. Figure 7 illustrates the relative pEPSP slopes, with the first stimulation pulse in TBS plotted with the burst phases. The relative pEPSP slopes at the CA3 and CA1 synapses were not significantly dependent upon the burst phases of CIBO (Fig. 7; one-way repeated measures ANOVA; $n=3$ each at the CA3 and CA1 synapses; $\mathrm{p}>0.05)$. The correlation between the relative slope of the first pEPSP in TBS and the magnitude of LTP was analyzed. The Spearman's rank correlation coefficients at the CA3 and CA1 synapses in CIBO were 0.049 and 


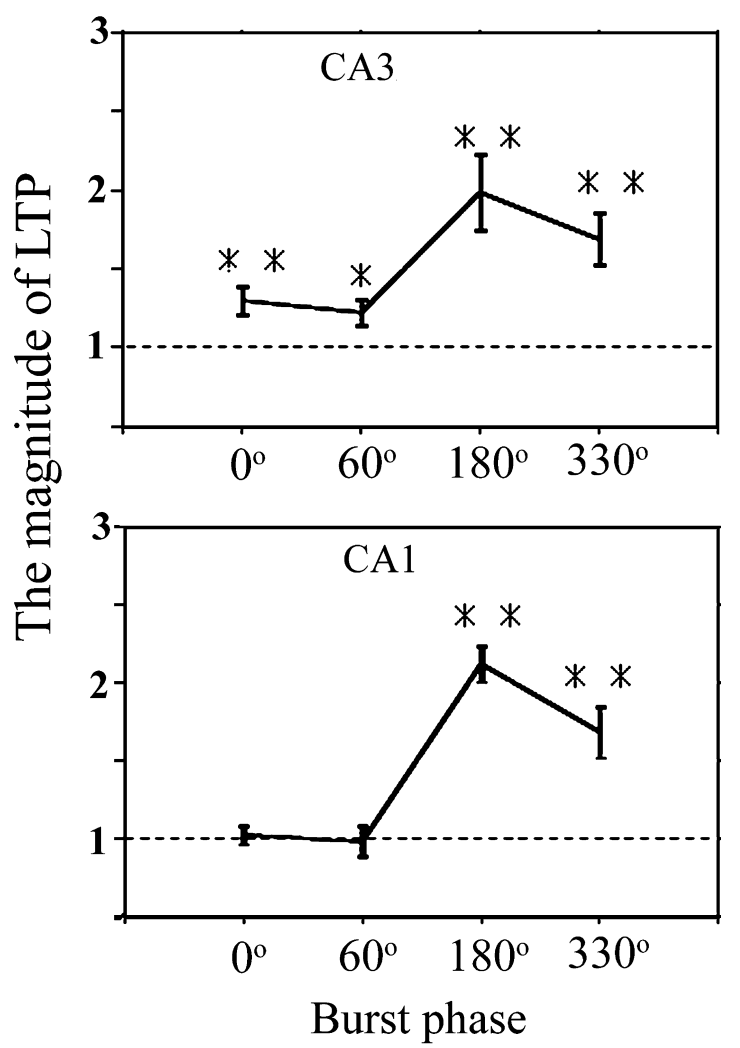

Figure 5 Magnitude of LTP at the CA3 (top) and CA1 (bottom) synapses with TBS depended on the burst phase of CIBO. These figures were constructed from the data in Figure 4. The relative pEPSP slopes at 40 min after TBS were compared with those at -1 min. ${ }^{*} p<0.05$ and ${ }^{* *} p<0.01$.

-0.063 , respectively (data not shown). There was no significance in any correlation (significant test for Spearman's rank correlation coefficient; $n=22$ and 16 at the CA3 and CA1 synapses in CIBO). Thus, the increases in the magnitude of LTP at the CA3 synapse and the induction of LTP at the CA1 synapse at the late burst phases of $180^{\circ}$ and $330^{\circ}$ were not dependent upon the degree of the excitatory postsynaptic potential.

\section{Discussion}

TBS was administered at several burst phases of CIBO at hippocampal CA3 and CA1 synapses. LTP was induced at all of the burst phases at the CA3 synapse, and the magnitude of LTP increased at the late burst phases of CIBO (Figs. 4 and 5). At the CA1 synapse, on the other hand, LTP was induced only at the late burst phases, while LTP was not induced at the earlier phases (Figs. 4 and 5). The modulation of the magnitude of LTP at both synapses was suppressed with the suppression of CIBO (Fig. 6). It was not due to a floor effect enabling the pEPSP slope to be changed by decreasing the maximal slope. CIBO is a model for in vivo $\beta$ rhythm, which occurs intermittently as bursts in a wax-and-wane fashion. The results from the present study suggest that 1) the generation of $\beta$ rhythm in vivo can modulate the synaptic plasticity at both CA 3 and CA1 synapses temporally; 2) the magnitude of LTP will be facilitated at the second half of the interval of the bursts of $\beta$ rhythm at the CA3 synapse; and 3) there is a time window for the induction of LTP at the second half of the interval of the bursts of $\beta$ rhythm at the CA1 synapse. With these data, the dependence of LTP induction on the burst phases of CIBO is demonstrated for the first time.

Carbachol activates muscarinic and nicotinic acetylcholine receptors. The induction of $\mathrm{CIBO}$ requires the activation of muscarinic acetylcholine receptors, whereas it does not require the activation of nicotinic acetylcholine receptors ${ }^{16}$. The magnitude of LTP was modulated in the inter-burst interval of CIBO (Fig. 5). Hence, the dependence of LTP induction on the burst phases of CIBO will not require the activation of nicotinic receptors.

The magnitude of LTP at the CA3 and CA1 synapses was increased at the late burst phases of CIBO (Figs. 4 and 5). Because the magnitude of LTP is dependent on excitatory synaptic transmission ${ }^{21,22}$, the pEPSP slopes were measured at the different burst phases. The slopes did not vary according to phase (Fig. 7). In the case of carbachol-induced $\theta$ oscillation $^{12}$, the least feedback inhibition occurs during the oscillation $^{23}$. Feedback inhibition to the CA3 and CA1 pyramidal cells is mediated by GABA transmission ${ }^{24}$ and the blockade of GABA transmission facilitates $\mathrm{LTP}^{25}$. Thus, the decrease in GABA transmission during the $\theta$ oscillation can increase the magnitude of $\mathrm{LTP}^{23}$. The increases in the magnitude of LTP at the late burst phases in the present study were not caused by excitatory synaptic transmission; they may have been caused by the disinhibition at the burst phases in CIBO, as is seen in the $\theta$ oscillation. In the interburst interval, GABA transmission can be modulated, and this modulation can cause a change in the magnitude of LTP.

LTP is facilitated to a greater degree when it is induced at the late burst phases, at the rest of CIBO (Figs. 4 and 5). It is facilitated, on the other hand, when it is induced during the generation of carbachol-induced $\theta$ oscillation. The modulation of the magnitude of LTP during the intermittent burst interval of $\theta$ oscillation is different from that of $\beta$ oscillation. The modulation can be dependent on the burst frequency. In vitro $\mathrm{CIBO}$ and $\theta$ oscillation are models for in vivo $\beta$ and $\theta$ rhythm, respectively. The difference of the two in vivo rhythms is not only the frequency, but also the induction time window for LTP relative to the onset of the burst of the rhythm.

Both hippocampal $\theta$ and $\beta$ rhythms are induced when a rodent processes spatial information in a novel environment. When the rodent is placed in a novel environment and begins to explore, hippocampal $\theta$ rhythm is induced. Initially, $\beta$ rhythm is not observed; $\beta$ rhythm is induced when the rodent revisits a place it experienced before ${ }^{2}$. Hippocampal $\theta$ rhythm is thought to be related to spatial memory 


\section{A}
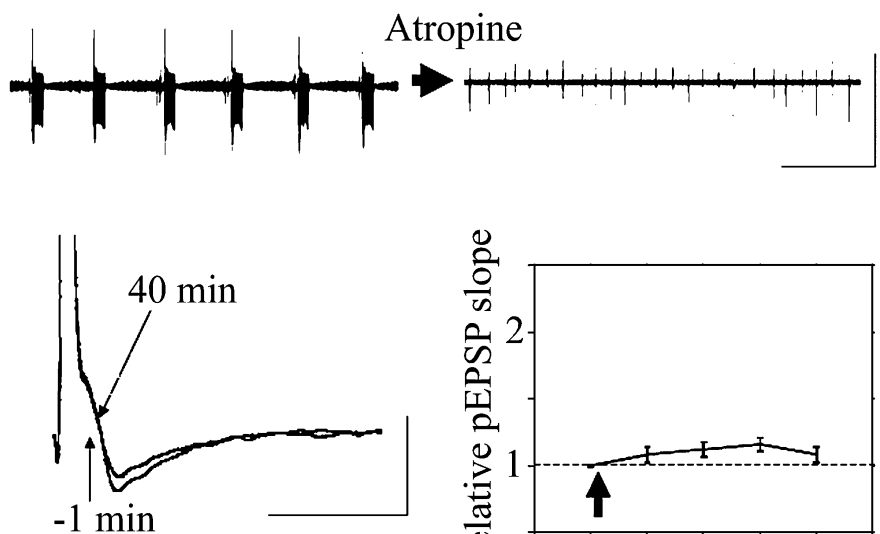

B
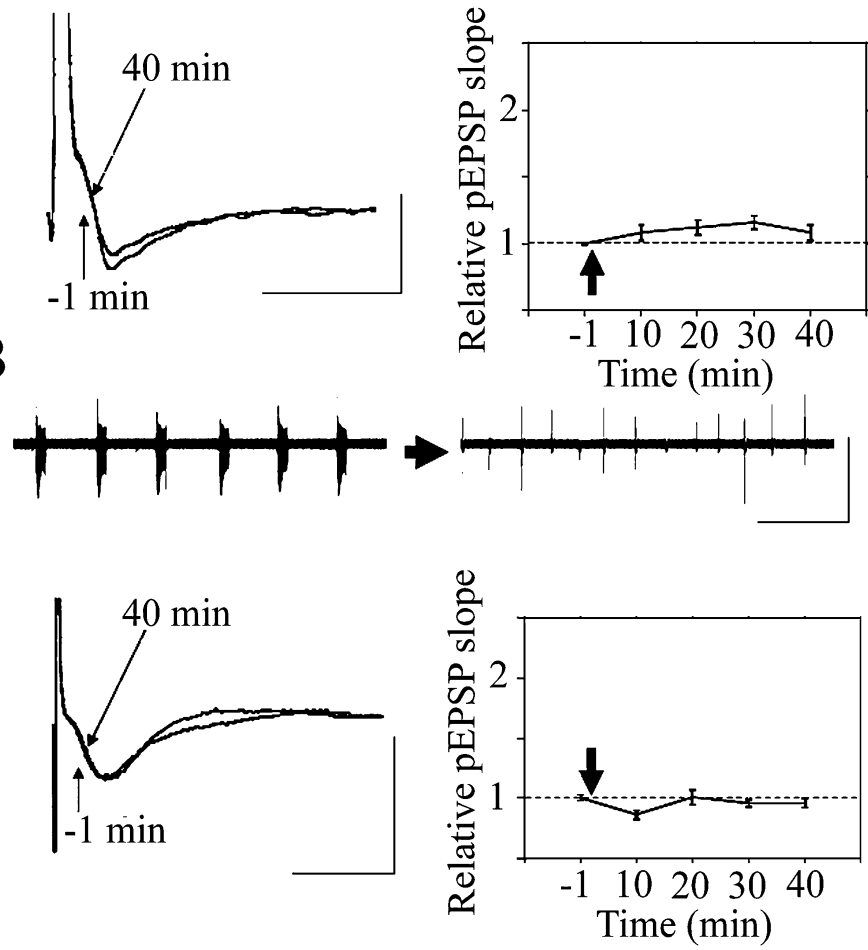

Figure 6 The effect of atropine on CIBO and on LTP induced by CIBO. (A) Atropine suppressed CA3 CIBO (top), and suppressed the induction of LTP at CA3 synapse (bottom). The time course of the relative pEPSP slope is shown in the bottom right figure. The thick arrow indicates the onset of TBS. Scale bars; $50 \mathrm{~s}$ and $4 \mathrm{mV}$, and $20 \mathrm{~ms}$ and $2 \mathrm{mV}$ in the top and bottom figures, respectively. (B) Atropine also suppressed CA1 CIBO (top), and LTP at the CA1 synapse (bottom). Scale bars; $50 \mathrm{~s}$ and $2 \mathrm{mV}$, and $20 \mathrm{~ms}$ and $3 \mathrm{mV}$ in the top and bottom figures, respectively. The data in (A) and (B) were obtained from 3 hippocampal slices each.

encoding and retrieval of the stored representation ${ }^{8,26} . \beta$ rhythm is thought to contribute to cued recall by comparing internal representations with incoming sensory inputs and finding a matching representation of the inputs ${ }^{3}$. When a rodent is first placed into a novel environment, sensory inputs indicating place information from the entorhinal cortex are encoded in the recurrent connections of hippocampal CA3 pyramidal cells ${ }^{7,27}$. While the rodent is exploring the environment, it encodes incoming inputs as new representations of the places, one by one. Subsequently, when the rodent revisits the same place as before, the hippocampal CA1 network compares the stored representation retrieved in CA3 with the sensory inputs of the current place, and engages in the match/mismatch process ${ }^{3,28,29}$. In the process, the CA1 region discards the mismatched representation until it finds a proper representation matching the current position of the rodent. When the CA1 region engages in the match process, induction of LTP at the CA1 synapse is necessary to build an association between the matched repre- sentation with the current sensory inputs ${ }^{3,8,30}$. The results of this study show that the time window for LTP induction at the CA1 synapse would be determined by the burst of $\beta$ rhythm. Thus, there will be a temporal relationship between the hippocampal match process and the generation of $\beta$ rhythm in spatial processing. The timing of matching the retrieved representation may be determined by the bursts of $\beta$ rhythm. During the generation of CIBO, on the other hand, LTP was not induced (Figs. 4 and 5). Managing the mismatch representations does not require LTP $^{3}$. Thus, during $\beta$ rhythm, the mismatch process may be completed. The in vivo inter-burst intervals of $\beta$ rhythm fluctuate ${ }^{2}$ different from the in vitro CIBO model. The different duration for the match and mismatch process in case of in vivo may reflect the fluctuation of the interval. When the CA1 region detects a mismatched representation, it sends a signal that resets the retrieving representation to the $\mathrm{CA} 3$ region, prompting it to induce another representation and send it back to the CA1 region $^{7,8}$. 


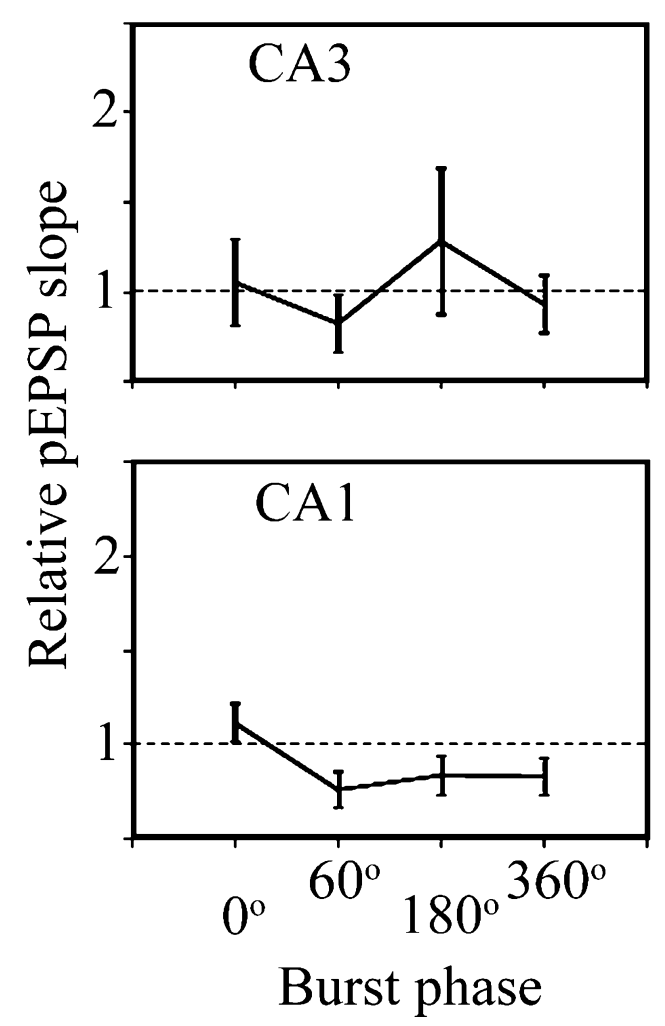

Figure 7 The dependency of the relative pEPSP slopes on the burst phases at the CA1 and CA3 synapses in CIBO. pEPSP slopes in CIBO are not dependent upon the burst phase of CIBO.

The hippocampal CA3 region is crucial for memory encoding and retrieval ${ }^{26,31}$. It retrieves stored representations and sends them to the CA1 region during the match/mismatch process. In spatial processing, the CA3 network recalls the representation of a place that the rodent previously experienced, and has it propagate to the CA1 region; when the representation matches with the sensory input in CA1 region, and the representation accompanies memory reconsolidation ${ }^{32}$. This reconsolidation accompanies synaptic plasticity $^{33}$. As discussed above, the match process is completed relative to the bursts of $\beta$ rhythm in the CA1 region. As evidenced by the present results, at the CA3 synapse, the largest magnitude of LTP was temporally related to match process. It is consistent that LTP is facilitated in the match process to reconsolidate the matched representation of the current place in the $\mathrm{CA} 3$ region. The long-range synchronization $^{6}$ of $\beta$ rhythm between the CA3 and CA1 regions may be necessary for perfecting the timing between representation retrieval in the $\mathrm{CA} 3$ region and the match/ mismatch process in the $\mathrm{CA} 1$ region.

\section{Conclusion}

Hippocampal $\beta$ rhythm occurs intermittently as bursts. LTP is induced at various burst phases of CIBO at the CA3 and CA1 synapses. At the CA3 synapse, LTP was induced at all burst phases and the magnitude of LTP increased at the late burst phases of CIBO. At the CA1 synapse, LTP was induced only during at the late burst phases. The modulation of LTP was disrupted at both synapses when CIBO was blocked. These results suggest that the intermittent bursts of $\beta$ rhythm may determine the temporal period for synaptic change at the $\mathrm{CA} 3$ and $\mathrm{CA} 1$ subregions in the hippocampal neuronal network. These results may reflect the coordinated memory processes of the CA3 and CA1 regions for stored representation retrieval and the match/mismatch process.

\section{Acknowledgements}

We thank Prof. Satoru Ishizuka and Prof. Katsumi Tateno of Kyushu Institute of Technology in Fukuoka, Japan for a fruitful discussion. We also thank the two anonymous reviewers for their helpful suggestions.

This work was supported by a Grant-in-Aid for Scientific Research on Innovative Areas "Neural creativity for communication (No.4103)" (24120712) of MEXT, Japan.

\section{References}

1. Bland, B.H. The physiology and pharmacology of hippocampal formation theta rhythms. Prog. Neurobiol. 26, 1-54 (1986).

2. Berke, J. D., Hetrick, V., Breck, J. \& Greene, R. W. Transient $23-30 \mathrm{~Hz}$ oscillations in mouse hippocampus during exploration of novel environments. Hippocampus 18, 519-529 (2008).

3. Grossberg, S. Beta oscillations and hippocampal place cell learning during exploration of novel environments. Hippocampus 19, 881-885 (2009).

4. Kay, L. M., Beshel, J., Brea, J., Martin, C., Rojas-Libano, D. \& Kopell, N. Olfactory oscillations: the what, how and what for. Trends Neurosci. 32, 207-214 (2009).

5. Martin, C., Beshel, J. \& Kay, L. M. An olfacto-hippocampal network is dynamically involved in odor-discrimination learning. J. Neurophysiol. 98, 2196-2205 (2007).

6. Bibbig, A., Traub, R.D. \& Whittington, M. A. Long-range synchronization of gamma and beta oscillations and the plasticity of excitatory and inhibitory synapses: a network model. J. Neurophysiol. 88, 1634-1654 (2002).

7. Hasselmo, M.E. \& Wyble, B.P. Free recall and recognition in a network model of the hippocampus: simulating effects of scopolamine on human memory function. Behav. Brain Res. 89, 1-34 (1997).

8. Lisman, J. E. \& Otmakhova, N. A. Storage, recall, and novelty detection of sequences by the hippocampus: elaborating on the SOCRATIC model to account for normal and aberrant effects of dopamine. Hippocampus 11, 551-568 (2001).

9. Holscher, C., Anwyl, R. \& Rowan, M. J. Stimulation on the positive phase of hippocampal theta rhythm induces longterm potentiation that can be depotentiated by stimulation on the negative phase in area CA1 in vivo. J. Neurosci. 17, 6470-6477 (1997).

10. Konopacki, J., Maciver, M.B., Bland, B.H. \& Roth, S.H. Theta in hippocampal slices: relation to synaptic responses of dentate neurons. Brain Res. Bull. 18, 25-27 (1987).

11. Williams, J.H. \& Kauer, J. A. Properties of carbachol-induced oscillatory activity in rat hippocampus. J. Neurophysiol. 78, 2631-2640 (1997). 
12. Natsume, K. \& Kometani, K. Theta-activity-dependent and -independent muscarinic facilitation of long-term potentiation in guinea pig hippocampal slices. Neurosci. Res. 27, 335-341 (1997).

13. Amaral, D. G. \& Witter, M.P. The three-dimensional organization of the hippocampal formation: a review of anatomical data. Neuroscience 31, 571-591 (1989).

14. Holscher, C. Long-term potentiation: a good model for learning and memory? Prog Neuropsychopharmacol. Biol. Psychiatry. 21, 47-68 (1997).

15. Hasselmo, M. E., Schnell, E. \& Barkai, E. Dynamics of learning and recall at excitatory recurrent synapses and cholinergic modulation in rat hippocampal region CA3. J. Neurosci. 15, 5249-5262 (1995).

16. Arai, J. \& Natsume, K. The properties of carbachol-induced beta oscillation in rat hippocampal slices. Neurosci. Res. 54, 95-103 (2006).

17. Shimono, K., Brucher, F., Granger, R., Lynch, G. \& Taketani, M. Origins and distribution of cholinergically induced beta rhythms in hippocampal slices. J. Neurosci. 20, 8462-8473 (2000).

18. Hasselmo, M.E. \& Schnell, E. Laminar selectivity of the cholinergic suppression of synaptic transmission in rat hippocampal region CA1: computational modeling and brain slice physiology. J. Neurosci. 14, 3898-3914 (1994).

19. Kremin, T. \& Hasselmo, M.E. Cholinergic suppression of glutamatergic synaptic transmission in hippocampal region CA3 exhibits laminar selectivity: Implication for hippocampal network dynamics. Neuroscience 149, 760-767 (2007).

20. Wyble, B.P., Linster, C. \& Hasselmo, M.E. Size of CA1evoked synaptic potentials is related to theta rhythm phase in rat hippocampus. J. Neurophysiol. 83, 2138-2144 (2000).

21. Artola, A. \& Singer, W. Long-term depression of excitatory synaptic transmission and its relationship to long-term potentiation. Trends Neurosci. 16, 480-487 (1993).

22. Bear, M.F. Mechanism for a sliding synaptic modification threshold. Neuron 15, 1-4 (1995).
23. Natsume, K. \& Kometani, K. Suppression of pair-pulse depression of the population spike in the dentate gyrus during carbachol-induced theta-like activity in guinea pig hippocampal slices. Neurosci. Res. 29, 113-120 (1997).

24. Freund, T. F. \& Buzsaki, G. Interneurons of the hippocampus. Hippocampus 6, 347-470 (1996).

25. Wigstrom, H. \& Gustafsson, B. Facilitated induction of hippocampal long-lasting potentiation during blockade of inhibition. Nature 301, 603-604 (1983).

26. Kunec, S., Hasselmo, M.E. \& Kopell, N. Encoding and retrieval in the CA3 region of the hippocampus: a model of theta-phase separation. J. Neurophysiol. 94, 70-82 (2005).

27. Treves, A. \& Rolls, E. T. Computational constraints suggest the need for two distinct input systems to the hippocampal CA3 network. Hippocampus 2, 189-199 (1992).

28. Duncan, K., Ketz, N., Inati, S. J. \& Davachi, L. Evidence for area CA1 as a match/mismatch detector: a high-resolution fMRI study of the human hippocampus. Hippocampus 22, 389-398 (2012).

29. Hasselmo, M.E. The role of hippocampal regions CA3 and CA1 in matching entorhinal input with retrieval of associations between objects and context: theoretical comment on Lee et al. (2005). Behav. Neurosci. 119, 342-345 (2005).

30. Kumaran, D. \& Maguire, E. A. Which computational mechanisms operate in the hippocampus during novelty detection? Hippocampus 17, 735-748 (2007).

31. Gilbert, P.E. \& Brushfield, A.M. The role of the CA3 hippocampal subregion in spatial memory: a process oriented behavioral assessment. Prog. Neuropsychopharmacol. Biol. Psychiatry. 33, 774-781 (2009).

32. Nader, K., Schafe, G.E. \& Le Doux, J.E. Fear memories require protein synthesis in the amygdala for reconsolidation after retrieval. Nature 406, 722-726 (2000).

33. Lee, J. L. Memory reconsolidation mediates the updating of hippocampal memory content. Front. Behav. Neurosci. 4, 168 (2010). 